\title{
$r$-IDEALS IN $\Gamma$-INCLINE
}

\author{
M. MURALI KRISHNA RAO ${ }^{1, *}$, B. VENKATESWARLU ${ }^{2}$, NOORBHASHA RAFI ${ }^{3}$ \\ ${ }^{1}$ Department of Mathematics, GIT, GITAM University, Visakhapatnam- 530 045, Andhra Pradesh, India \\ ${ }^{2}$ Department of Mathematics, GST, GITAM University, Doddaballapura - 561 203, Bengaluru North, Karnataka, India \\ ${ }^{3}$ Department of Mathematics, Bapatla Engineering College, Bapatla-522 101, Andhra Pradesh, India \\ *Corresponding author: mmarapureddy@gmail.com \\ Received Oct. 28, 2018
}

\begin{abstract}
AвSTRACt. In this paper, we introduce the notion of ideal, $k$-ideal, $r$-ideal in $\Gamma$-incline. We study the properties of $r$-ideals and $k$-ideals in $\Gamma$-incline and relations between them.

2010 Mathematics Subject Classification.16A09, 06F25.
\end{abstract}

Key words and phrases: $\Gamma$-incline; incline; regular $\Gamma$-incline; $r$-ideal.

\section{INTRODUCTION}

The non trivial example of semiring first appeared in the work of German mathematician Richard Dedikind in 1894 in connection with the algebra of ideals of a commutative ring. The notion of semiring was introduced by American mathematician Vandiver [23] in 1934. Semiring is a well known universal algebra. Semirings have been used for studying optimization theory, graph theory, matrices, determinants, theory of automata, coding theory, analysis of computer programmes, etc.

The concept of incline was first introduced by Cao et al. [3] in 1984. Inclines are additively idempotent semirings in which products are less than or equal to either factor. Products reduce the values of quantities and make them go down which is why the structures were named inclines. Idempotent semirings and Kleene algebras have recently been established as fundamental structures in computer sciences. An incline is a generalization of Boolean algebra, fuzzy algebra and distributive lattice. An incline has both semiring structure and the poset structure. Every distributive lattice and every Boolean algebra is an incline but an incline need not be a distributive lattice. Set of all idempotent elements in an incline is a distributive lattice. Yao and Han studied the relations between ideals, filters and congruences in inclines and it is shown that there is a one to one correspondence between the set of ideals and the set of all regular congruences. Cao et al. [3] studied the incline and its applications. Kim and Rowsh have studied matrices over an incline. Many research scholars have been researched the theory of incline matrices. Few research scholars studied the algebraic structure of incline. inclines and Matrices over inclines are useful tools in diverse areas such as automata theory, design of switching 
circuits, graph theory, information systems, modeling, decision making, dynamical programming, control theory, classical and non classical path finding problems in graphs, fuzzy set theory, data analysis, medical diagnosis, nervous system, probable reasoning, physical measurement and so on. Ahn, Jun and Kim [1,2] studied ideals in incline and quotient incline. Ideals play an important role in advance studies and uses of algebraic structures. Generalization of ideals in algebraic structures is necessary for further study of algebraic structures. Many mathematicians proved important results and characterization of algebraic structures by using the concept and the properties of generalization of ideals in algebraic structures. The notion of ideals was introduced by Dedekind for the theory of algebraic numbers, was generalized by Noether for associative rings. The one and two sided ideals introduced by her, are still central concepts in ring theory and the notion of a one sided ideal of any algebraic structure is a generalization of notion of an ideal. In 1952, the concept of bi-ideals was introduced by Good and Hughes [5] for semigroups. The notion of bi-ideals in rings was introduced by Lajos and Szasz [8]. Quasi ideals are generalization of right ideals and left ideals whereas bi-ideals are generalization of quasi ideals.

In 1956, Steinfeld [22] first introduced the notion of quasi ideals for semigroups and then for rings. Iseki [7] introduced the concept of quasi ideal for a semiring. Murali Krishna Rao [17] introduced the concept of biinterior ideal for a semigroup. Henriksen [6] studied ideals in semirings with commutative addition. The notion of $\Gamma$-ring was introduced by Nobusawa [20] as a generalization of ring in 1964. Sen [21] introduced the notion of $\Gamma$-semigroup in 1981. The notion of ternary algebraic system was introduced by Lehmer [9] in 1932, Lister introduced ternary ring. Dutta and Kar [4] introduced the notion of ternary semiring which is a generalization of ternary ring and semiring. In 1995, Murali Krishna Rao [10-12] introduced the notion of $\Gamma$-semiring which is a generalization of $\Gamma$-ring, ternary semiring and semiring. After the paper [10] was published, many mathematicians obtained interesting results on $\Gamma$-semirings. Murali Krishna Rao and Venkateswarlu [18] introduced the notion of regular $\Gamma$-incline and field $\Gamma$-semiring.

In this paper, we introduce the notion of ideal, and , $r$-ideal in $\Gamma$-incline. We study the properties of $r-$ ideals and $k$-ideals in $\Gamma$-incline and relations between them.

\section{Preliminaries}

In this section we will recall some of the fundamental concepts and definitions which are necessary for this paper.

Definition 2.1. A commutative incline $M$ with additive identity 0 and multiplicative identity 1 is a non-empty set $M$ with operations addition (+) and multiplication (.) defined on $M \times M \rightarrow M$ such that satisfying the following conditions for all $x, y, z \in M$

(i) $x+y=y+x$

(ii) $x+x=x$

(iii) $x+x y=x$

(iv) $y+x y=y$

(v) $x+(y+z)=(x+y)+z$

(vi) $x(y z)=x(y z)$

(vii) $x(y+z)=x y+x z$ 
(viii) $(x+y) z=x z+y z$

(ix) $x 1=1 x=x$

(x) $x+0=0+x=x$

(xi) $x y=y x$.

Definition 2.2. Let $(M,+)$ and $(\Gamma,+)$ be commutative semigroups. Then we call $M$ as a $\Gamma$-semiring, if there exists a mapping $M \times \Gamma \times M \rightarrow M$ is written $(x, \alpha, y)$ as $x \alpha y$ such that it satisfies the following axioms for all $x, y, z \in M$ and $\alpha, \beta \in \Gamma$

(i) $x \alpha(y+z)=x \alpha y+x \alpha z$

(ii) $(x+y) \alpha z=x \alpha z+y \alpha z$

(iii) $x(\alpha+\beta) y=x \alpha y+x \beta y$

(iv) $x \alpha(y \beta z)=(x \alpha y) \beta z$.

Every semiring $R$ is a $\Gamma$-semiring with $\Gamma=R$ and ternary operation $x \gamma y$ as the usual semiring multiplication. Definition 2.3. Let $(M,+)$ and $(\Gamma,+)$ be commutative semigroups. If there exists a mapping $M \times \Gamma \times M \rightarrow$ $M((x, \alpha, y) \rightarrow x \alpha y)$ such that it satisfies the following axioms for all $x, y, z \in M$ and $\alpha, \beta \in \Gamma$

(i) $x \alpha(y+z)=x \alpha y+x \alpha z$

(ii) $(x+y) \alpha z=x \alpha z+y \alpha z$

(iii) $x(\alpha+\beta) y=x \alpha y+x \beta y$

(iv) $x \alpha(y \beta z)=(x \alpha y) \beta z$

(v) $x+x=x$

(vi) $x+x \alpha y=x$

(vii) $y+x \alpha y=y$.

Then $M$ is called a $\Gamma$-incline.

Every incline $M$ is a $\Gamma$-incline with $\Gamma=M$ and ternary operation $x \gamma y$ as the usual incline multiplication.

In a $\Gamma$-incline we define the order relation such that for all $x, y \in M, y \leq x$ if and only if $y+x=x$. Obviously $\leq$ is a partially order relation over $M$.

Definition 2.4. A $\Gamma$-incline $M$ is said to have zero element if there exists an element $0 \in M$ such that $0+x=$ $x=x+0$ and $0 \alpha x=x \alpha 0=0$, for all $x \in M$.

Definition 2.5. A $\Gamma$-incline $M$ is said to be commutative $\Gamma$-incline if $x \alpha y=y \alpha x$, for all $x, y \in M$.

Definition 2.6. A $\Gamma$-subincline $I$ of a $\Gamma$-incline $M$ is a non-empty subset of $M$ which is closed under the $\Gamma$-incline operations addition and ternary operation.

Definition 2.7. An element $a \in M$ is said to be idempotent of $M$ if there exists $\alpha \in \Gamma$ such that $a=a \alpha a$ and $a$ is also said to be $\alpha$-idempotent.

Definition 2.8. If every element of $M$ is an idempotent of $M$, then $M$ is said to be idempotent $\Gamma$-incline $M$.

Definition 2.9. If every element of $M$ is a regular element of $M$, then $M$ is said to be a regular $\Gamma$-incline $M$. 
Definition 2.10. If $x \leq y$, for all $y \in M$, then $x$ is called the least element of $M$ and denoted as 0 . If $x \geq y$, for all $y \in M$ then $x$ is called the greatest element of $M$ and denoted as 1 .

Definition 2.11. A $\Gamma$-incline $M$ is said to be linearly ordered if $x, y \in M$ then either $x \leq y$ or $y \leq x$, where $\leq$ is an incline order relation.

Definition 2.12. An element $1 \in M$ is said to be unity if for each $x \in M$ there exists $\alpha \in \Gamma$ such that $x \alpha 1=$ $1 \alpha x=x$.

Definition 2.13. In a $\Gamma$-incline $M$ with unity 1 , an element $a \in M$ is said to be invertible if there exist $b \in M, \alpha \in \Gamma$ such that $a \alpha b=b \alpha a=1$.

Definition 2.14. A non- zero element $a$ in a $\Gamma$-incline $M$ is said to be a zero divisor if there exists non zero element $b \in M, \alpha \in \Gamma$ such that $a \alpha b=b \alpha a=0$.

Definition 2.15. A $\Gamma$-incline $M$ is said to be field $\Gamma$-incline if $M$ is a commutative $\Gamma$-incline with unity 1 and every non-zero element of $M$ is invertible.

Definition 2.16. A $\Gamma$-incline $M$ with unity 1 and zero element 0 is called an integral $\Gamma$-incline if it has no zero divisors.

Definition 2.17. A $\Gamma$-incline $M$ with zero element 0 is said to be hold cancelation laws if $a \neq 0, a \alpha b=a \alpha c, b \alpha a=$ $c \alpha a$, where $a, b, c \in M, \alpha \in \Gamma$ then $b=c$.

Definition 2.18. Let $M$ be a $\Gamma$-incline with unity 1 and zero element 0 . Then $M$ is said to be pre -integral incline if $M$ holds cancelation law.

Definition 2.19. A $\Gamma$-incline $M$ is said to be zero sum free $\Gamma$-incline if $x+y=0$, then $x=0$ and $y=$ 0 , for all $x, y \in M$.

Example 2.20. Let $S$ be a semiring and $M_{p, q}(S)$ denote the additive abelian semigroup of all $p \times q$ matrices with identity element whose entries are from $S$. Then $M_{p, q}(S)$ is a $\Gamma$-semiring with $\Gamma=M_{p, q}(S)$ ternary operation is defined by $x \alpha z=x\left(\alpha^{t}\right) z$ as the usual matrix multiplication, where $\alpha^{t}$ denote the transpose of the matrix $\alpha$, for all $x, y$ and $\alpha \in M_{p, q}(S)$.

Example 2.21. If $M=[0,1]$ and $\Gamma=N$, a binary operation + is defined as $a+b=\max \{a, b\}$ and ternary operation is defined as $x r y=\min \{x, r, y\}$, for all $x, y \in M, r \in \Gamma$ then $M$ is a $\Gamma$-incline .

Example 2.22. If $M=[0,1], \Gamma=\{0,1\}$, binary operation + is maximum, ternary operation $a \alpha b$ is the usual multiplication for all $a, b \in M, \alpha \in \Gamma$ then $M$ is $\Gamma$-incline with unity 1 .

Definition 2.23. Let $M$ and $N$ be $\Gamma$-inclines. A mapping $f: M \rightarrow N$ is called a homomorphism if

(i) $f(a+b)=f(a)+f(b)$

(ii) $f(a \alpha b)=f(a) \alpha f(b)$, for all $a, b \in M, \alpha \in \Gamma$. 


\section{3. $r$-IDEALS AND $k$-IDEALS IN $\Gamma$-INCLINE}

In this section, we introduce the notion of ideal, $r$-ideal, $k$-ideal in $\Gamma$-incline. We study their properties in $\Gamma$-incline and relations between them.

Definition 3.1. A $\Gamma$-subincline $I$ of a $\Gamma$-incline $M$ is called an ideal if it is a lower set. i.e., for any $x \in I, y \in M$ and $y \leq x \Rightarrow y \in I$.

Definition 3.2. A proper ideal $P$ of a $\Gamma$-incline $M$ is said to be prime ideal if for all $x, y \in M, \alpha \in \Gamma, x \alpha y \in P \Rightarrow$ $x \in P$ or $y \in P$.

Definition 3.3. A $\Gamma$-subincline $I$ of a $\Gamma$-incline $M$ is said to be a left (right) $r$-ideal of $M$ if $M \Gamma I \subseteq I(I \Gamma M \subseteq I)$.

Definition 3.4. If $I$ is both a left $r$-ideal and a right $r$-ideal, then $I$ is called a $r$-ideal of $\Gamma$-incline $M$.

Definition 3.5. An ideal $K$ of a $\Gamma$-incline $M$ is said to be maximal ideal if $K \neq M$ and for every ideal $I$ of $M$ with $K \subseteq I \subseteq M$, then either $I=K$ or $I=M$.

Definition 3.6. A $\Gamma$-subincline $I$ of a $\Gamma$-incline $M$ is said to be $k$-ideal if $x \in M, x+y \in I, y \in I, x \in M$ then $x \in I$.

Definition 3.7. A $\Gamma$-incline $M$ is said to be $r$-simple if it has no proper $r$-ideals of $M$.

Theorem 3.8. Let $I$ be $a \Gamma$-subincline of $a \Gamma$-incline $M$. Then $I$ is an ideal of $M$ if and only if $I$ is a $k$-ideal of $M$.

Proof. Let $I$ be an ideal of the $\Gamma$-incline $M$ and $x \in M, x+y \in I, y \in I$.

$$
\begin{aligned}
x+y & =(x+x)+y \\
& =x+(x+y) \\
\Rightarrow x & \leq x+y .
\end{aligned}
$$

Therefore, by definition of an ideal, $x \in I$. Hence $I$ is a $k$-ideal of $M$.

Conversely suppose that $I$ is a $k$-ideal of the $\Gamma$-incline $M$. Let $y \in M, x \in I$ and $y \leq x$.

$$
\begin{aligned}
& \Rightarrow y+x=x \\
& \Rightarrow y+x \in I \\
& \Rightarrow y \in I, \text { since } I \text { is a } k \text {-ideal of } \Gamma \text {-incline } M .
\end{aligned}
$$

Hence $I$ is an ideal of the $\Gamma$-incline $M$.

Theorem 3.9. If $I$ is a $r$-ideal of an idempotent $\Gamma$-incline $M$, then $I$ is a $k$-ideal of $M$. 
Proof. Suppose $x+y \in I, y \in I, x \in M$. Then $x+x \beta y=x$, for all $\beta \in \Gamma$ and there exists $\alpha \in \Gamma$ such that $x \alpha x=x$.

We have $x+y \alpha x=x$. Since

$$
\begin{aligned}
& x+y \in I, \alpha \in \Gamma, x \in M \\
& (x+y) \alpha x \in I \\
\Rightarrow & x \alpha x+y \alpha x \in I \\
\Rightarrow & x+y \alpha x \in I \\
\Rightarrow & x \in I .
\end{aligned}
$$

Suppose $x \leq y, y \in I$. Then $x+y=y$. Therefore $x \in I$.

Hence $I$ is a $k$-ideal of the $\Gamma$-incline $M$.

Theorem 3.10. Let $M$ be $a \Gamma$-incline. If $I$ is an ideal of $a \Gamma$-incline $M$, then $I$ is a $r$-ideal of $M$.

Proof. Suppose $I$ is an ideal of the $\Gamma$-incline $M, x \in I, y \in M$ and $\alpha \in \Gamma$.

Then $x \alpha y \leq x$ and $y \alpha x \leq x$.

Since $I$ is an ideal, $x \alpha y, y \alpha x \in I$.

Hence $I$ is a $r$-ideal of the $\Gamma$-incline $M$.

The following example shows that converse of Theorem 3.10 need not be true.

Example 3.11. Let $I=[0,1]$ be a set of reals between 0 and 1 with $x+y=\max \{x, y\}$ and $x \cdot y=x y$, where $\cdot$ is a usual multiplication for all $x, y \in I$. Then $I$ is a incline.

Let $M$ be the set of all $2 \times 2$ matrices whose elements be in $I$ and $\Gamma=M$. Now we define

$$
A+B=\left(a_{i j}+b_{i j}\right) \text { and } A \times B=\left(a_{i j} c_{i j} b_{i j}\right)
$$

where $A=\left(a_{i j}\right), B=\left(b_{i j}\right)$ and $C=\left(c_{i j}\right)$ are in $M$. Then $M$ is a $\Gamma$-incline.

Let $B=\left\{\left(\begin{array}{ll}a & b \\ 0 & 0\end{array}\right) \mid a, b \in I\right\}$. Suppose $\left(\begin{array}{cc}a & b \\ 0 & 0\end{array}\right) \in B,\left(\begin{array}{cc}x & y \\ z & w\end{array}\right)\left(\begin{array}{ll}p & q \\ r & s\end{array}\right) \in M$.

Then $\left(\begin{array}{ll}a & b \\ 0 & 0\end{array}\right)\left(\begin{array}{cc}x & y \\ z & w\end{array}\right)\left(\begin{array}{cc}p & q \\ r & s\end{array}\right)=\left(\begin{array}{cc}a p x & b q y \\ 0 & 0\end{array}\right) \in B$.

Suppose $A=\left(a_{i j}\right)$ and $B=\left(b_{i j}\right) \in M$.

We define $A \leq B$ if and only if $a_{i j} \leq b_{i j}$, for all $i, j$.

We have $\left(\begin{array}{cc}0.5 & 0.5 \\ 0 & 0\end{array}\right) \leq\left(\begin{array}{cc}0.5 & 0.6 \\ 0 & 0\end{array}\right) \in B$ but $\left(\begin{array}{cc}0.5 & 0.5 \\ 0 & 0\end{array}\right) \notin B$.

Hence $B$ is a $r$-ideal but not an ideal of the $\Gamma$-incline $M$.

Theorem 3.12. Let $M$ be $a \Gamma$-incline with unity 1 and zero element 0 . If $I$ is a $r$-ideal containing a unit element, then $I=M$. 
Proof. Let $I$ be a $r$-ideal of the $\Gamma$-incline $M$ containing a unit element $u$ and $x \in M$. Then there exists $\alpha \in \Gamma$ such that $x \alpha 1=x$. Since $I$ is a $r$-ideal, $x \alpha u \in I$. and $u$ is a unit, there exist $\delta \in \Gamma, t \in M$ such that $u \delta t=1$.

$$
\begin{aligned}
& \Rightarrow x \alpha u \delta t=x \alpha 1=x \\
& \Rightarrow x \in I .
\end{aligned}
$$

Hence $I=M$.

Theorem 3.13. A field $\Gamma$-incline $M$ is $r$-simple.

Proof. Let $I$ be a proper $r$-ideal of the field $\Gamma$-incline $M$. Every non-zero element of $I$ is a unit. By Theorem 3.12, we have $I=M$. Hence field $\Gamma$-incline is $r$-simple.

Definition 3.14. Let $A$ be a non-empty subset of a $\Gamma$-incline $M$. Then the set $\{x \in M \mid a \alpha(a \alpha x)=0$, for all $\alpha \in$ $\Gamma$ and $a \in A\}$. It is denoted by $\operatorname{Annl}(A)$.

Theorem 3.15. Let $I$ be an ideal of $a \Gamma$-incline $M$. Then $I^{*}=\{x \in M \mid x+a \in I$, for some $a \in I, \alpha \in \Gamma\}$ is a $k$-ideal of $M$.

Proof. Let $x, y \in I^{*}$. Then there exist $a, b \in I$ such that $x+a, y+b \in I$ and

$$
\begin{aligned}
& (x+a)+(y+b)=x+y+a+b \in I \\
\Rightarrow & x+y \in I^{*} . \\
& (x+a) \alpha(y+b)=x \alpha y+x \alpha b+a \alpha y+a \alpha b \\
\Rightarrow & x \alpha y \in I^{*} .
\end{aligned}
$$

Therefore $I^{*}$ is a $\Gamma$-subincline of $M$.

Let $x, y \in M, x \leq y$ and $y \in I^{*}$. Then there exists $a \in I$ such that $y+a \in I$. We have

$$
\begin{aligned}
& x+y=y \\
\Rightarrow & x+y+a=y+a \\
\Rightarrow & x+y+a \in I \\
\Rightarrow & x \in I .
\end{aligned}
$$

Hence $I^{*}$ is a $k$-ideal of $M$.

Theorem 3.16. Let $A$ and $B$ be subsets of $a \Gamma$-incline $M$. If $A \subseteq B$ then $A n n l(B) \subseteq A n n l(A)$.

Proof. Let $x \in B$. Then

$$
\begin{aligned}
& b \alpha(b \alpha x)=0, \text { for all } \alpha \in \Gamma, b \in B \\
\Rightarrow & b \alpha(b \alpha x)=0, \text { for all } \alpha \in \Gamma, b \in A, \text { since } A \subseteq B \\
\Rightarrow & b \in \operatorname{Annl}(A) .
\end{aligned}
$$

Hence $A n n l(B) \subseteq A n n l(A)$. 
Definition 3.17. A mapping $f$ from a $\Gamma$-incline $M$ into a $\Gamma$-incline $N$ is said to be regular homomorphism if it satisfies the following

(i) $f(x+y)=f(x)+f(y)$

(ii) $f(x \alpha y)=f(x) \alpha f(y)$

(iii) $f(0)=0$,for all $x, y \in M, \alpha \in \Gamma$.

If the $\Gamma$-incline $M$ is additively cancellative, then any homomorphism is regular.

Theorem 3.18. Let $f: M \rightarrow N$ be a homomorphism of $\Gamma$-inclines $M$ and $N$. If $I$ is a left $r$-ideal of $M$, then $f(I)$ is a left $r$-ideal of $N$.

Proof. Let $x, y \in f(I), \alpha \in \Gamma$. Then there exist $a, b \in I$ such that $f(a)=x$ and $f(b)=y$. Then

$$
\begin{aligned}
x+y & =f(a)+f(b) \\
& =f(a+b) \in f(I) . \\
x \alpha y & =f(a) \alpha f(b) \\
& =f(a \alpha b) \in f(I) .
\end{aligned}
$$

Hence $f(I)$ is a $\Gamma$-subincline of $N$.

Let $x \in f(I), a \in N$ and $\alpha \in \Gamma$. Then there exist $y \in I$ and $b \in M$ such that $f(y)=x$ and $f(b)=a$. Therefore

$$
x \alpha a=f(y) \alpha f(b)=f(y \alpha b) \in f(I), \text { since } I \text { is a left } r \text { - ideal . }
$$

Hence $f(I)$ is a left $r$-ideal of $N$.

Theorem 3.19. Let $A$ be a non-empty subset of a commutative $\Gamma$-incline $M$. Then $A n n l(A)$ is an $r$-ideal of $M$.

Proof. Let $x, y \in \operatorname{Annl}(A), \alpha \in \Gamma$. Then

$$
\begin{aligned}
& a \alpha(a \alpha x)=0 \text { and } a \alpha(a \alpha y)=0 \\
\Rightarrow & a \alpha(a \alpha(x+y))=a \alpha(a \alpha x)+a \alpha(a \alpha y)=0+0=0 \\
\Rightarrow & x+y \in \operatorname{Annl}(A) . \\
& a \alpha(a \alpha(x \alpha y))=a \alpha(a \alpha x) \alpha y=0 \alpha y=0 \\
\Rightarrow & x \alpha y \in \operatorname{Annl}(A) .
\end{aligned}
$$

Hence $\operatorname{Annl}(A)$ is a $\Gamma$-subincline of $M$.

Suppose $x \in \operatorname{Annl}(A), y \in M$ and $\beta \in \Gamma$. Then

$$
a \alpha(a \alpha(x \beta y))=a \alpha(a \alpha x) \beta y=0 \beta y=0, \text { for all } \alpha \in \Gamma, a \in A .
$$

Therefore $x \beta y \in \operatorname{Annl}(A)$. Hence $\operatorname{Annl}(A)$ is a $r$-ideal of the $\Gamma$-incline $M$.

Theorem 3.20. Let $f: M \rightarrow N$ be a regular homomorphism of $\Gamma$-incline s $M$ and $N$ and $A$ be a subset of $M$ and $M$ is additively cancellative $\Gamma-$ incline. Then $f(\operatorname{Annl}(A)) \subseteq \operatorname{Annl}(f(A))$. 
Proof. Let $y \in f(\operatorname{Annl}(A))$ and $b \in[f(A)]$. Then there exist $x \in \operatorname{Annl}(A)$ and $a \in A$ such that $f(x)=y$ and $f(a)=b$.

$$
\begin{aligned}
b \alpha(b \alpha y) & =f(a) \alpha(f(a) \alpha f(x)) \\
& =f(a \alpha(a \alpha x)) \\
& =f(0) \\
& =0 .
\end{aligned}
$$

Hence $y \in \operatorname{Annl}(f(A))$. This complete the proof of the theorem.

Theorem 3.21. Let $f: M \rightarrow N$ be a homomorphism of $\Gamma$-inclines $M$ and $N$. If $J$ is a $r$-ideal of $N$ then $f^{-1}(J)$ is a $r$-ideal of $M$.

Proof. Let $x, y \in f^{-1}(J)$ and $\alpha \in \Gamma$. Then

$$
\begin{aligned}
& f(x+y)=f(x)+f(y) \in J, \text { since } J \text { is a sub incline } \\
& \Rightarrow x+y \in f^{-1}(J) . \\
& f(x \alpha y)=f(x) \alpha f(y) \in J, \text { since } J \text { is a sub incline. }
\end{aligned}
$$

Hence $f^{-1}(J)$ is a sub incline of $M$.

Let $x \in M, y \in f^{-1}(J)$. Then $f(y) \in J$.

$$
\begin{aligned}
& f(x \alpha y)=f(x) \alpha f(y) \in J, \text { since } J \text { is an } r \text {-ideal of } M \\
& f(y \alpha x)=f(y) \alpha f(x) \in J, \text { since } J \text { is an } r \text {-ideal of } M .
\end{aligned}
$$

Therefore $x \alpha y$ and $y \alpha x$ are in $f^{-1}(J)$. Hence $f^{-1}(J)$ is a $r$-ideal of $M$.

\section{Conclusion}

In this paper, we introduced the notion of $r$-ideal in $\Gamma$-incline. We study the properties of $r$-ideals and proved that if $I$ is an ideal of a $\Gamma$-incline $M$ then $I$ is a $r$-ideal of $M$.

\section{REFERENCES}

[1] S. S. Ahn, Y.B. Jun and H.S. Kim, Ideals and quotients of incline algebras, Comm. Korean Math. Soc., 16 (2001), $2573-583$.

[2] S. S. Ahn and H. S. Kim, On $r$-ideals in incline algebras, Comm. Korean Math. Soc., 17(2) (2002), 229-235.

[3] Z. Q. Cao, K. H. Kim and F. W. Roush, Incline algebra and applications, JohnWiley and Sons, New York, 1984.

[4] T.K. Dutta and S. Kar, On regular ternary semirings, in: K.P. Shum, Z.H. Wan and230 J.P. Zhang (Eds.), Advances in Algebra, Proceedings of the ICM Satellite Conference231 in Algebra and Related Topics (Singapore, World Scientific, 2003) 343-355.

[5] R. A. Good and D. R. Hughes, Associated groups for a semigroup, Bull. Am. Math.Soc., 58(1952), 624-625.

[6] M. Henriksen, Ideals in semirings with commutative addition, Amer. Math. Soc. Notices, 5 (1958), 321.

[7] K. Iseki, Quasi-ideals in semirings without zero, Proc. Japan Acad., 34 (1958), 79-84.

[8] S. Lajos and F. A. Szasz, On the bi-ideals in associative ring, Proc. Japan Acad., 46 (1970), 505-507.

[9] D.H. Lehmer, A ternary analogue of Abelian groups Am. J. Math. 59 (1932) 329-338.

[10] M. Murali Krishna Rao, $\Gamma$-semirings-I, Southeast Asian Bull. Math., 19 (1) (1995), 49-54.

[11] M. Murali Krishna Rao, $\Gamma$-semirings-II,Southeast Asian Bulletin of Mathematics 21(5) (1997), 281-287, 
[12] M. Murali Krishna Rao,The Jacobson Radical of a $\Gamma$-semiring,Southeast Asian Bulletin of Mathematics 23(1999),127-134

[13] M. Murali Krishna Rao, Left bi-quasi ideals of semirings, Bull. Int. Math. Virtual Inst., 8(2018), 45-53.

[14] Marapureddy Murali Krishna Rao, Bi-quasi-ideals and fuzzy bi-quasi ideals of $\Gamma$ - semigroups, Bull. Int. Math. Virtual Inst., 7(2)(2017), 231-242.

[15] M. Murali Krishna Rao, $\Gamma$ - semiring with identity, Discuss. Math. Gen. Algebra Appl., 37 (2017) 189-207,

[16] M. Murali Krishna Rao, Ideals in ordered $\Gamma$-semirings, Discuss. Math. Gen. Algebra Appl., 38 (2018), 47-68.

[17] M. Murali Krishna Rao, Bi-interior ideals in semigroups, Discuss. Math. Gen. Algebra Appl., 38 (2018), 69-78.

[18] M. Murali Krishna Rao, B. Venkateswarlu and N.Rafi, Left bi-quasi-ideals of $\Gamma$-semirings, Asia Pacific J. of Math., 4(2) (2017), 144-153.

[19] M. Murali Krishna Rao and B. Venkateswarlu, Regular $\Gamma$-incline and field $\Gamma$-semiring, Novi sad. J. Math., 45(2) 2015, $155-171$.

[20] N. Nobusawa, On a generalization of the ring theory, Osaka. J.Math., 1 (1964), 81 - 89.

[21] M. K. Sen, On $\Gamma$-semigroup, Proc. of Inter. Con. of Alg. and its Appl., Decker Publication, New York, (1981), 301-308.

[22] O. Steinfeld, Uher die quasi ideals, Von halbgruppend Publ. Math., Debrecen, 4 (1956), 262 - 275.

[23] H. S. Vandiver, Note on a simple type of algebra in which cancellation law of addition does not hold, Bull. Amer. Math. Soc.(N.S.), 40 (1934), 914-920. 